\title{
The Functional Renormalization Group Treatment of Disordered Systems, a Review
}

\author{
Kay Jörg Wiese \\ KITP, Kohn Hall, University of California at Santa Barbara, Santa Barbara, CA 93106, USA
}

Nov. 23, 2002

\begin{abstract}
We review current progress in the functional renormalization group treatment of disordered systems. After an elementary introduction into the phenomenology, we show why in the context of disordered systems a functional renormalization group treatment is necessary, contrary to pure systems, where renormalization of a single coupling constant is sufficient. This leads to a disorder distribution, which after a finite renomalization becomes non-analytic, thus overcoming the predictions of the seemingly exact dimensional reduction. We discuss, how a renormalizable field theory can be constructed, even beyond 1-loop order. We then discuss an elastic manifold imbedded in $N$ dimensions, and give the exact solution for $N \rightarrow \infty$. This is compared to predictions of the Gaussian replica variational ansatz, using replica symmetry breaking. We finally discuss depinning, both isotropic and anisotropic, and the scaling function for the width distribution of an interface.
\end{abstract}

\section{Contents}

1 Introduction $\quad 2$

2 Physical realizations, model and observables $r$

3 Treatment of disorder $r$

4 Dimensional reduction $\quad 4$

5 The Larkin-length $\quad 5$

6 The functional renormalization group (FRG) 5

$\begin{array}{lll}7 & \text { Why is a cusp necessary? } & 7\end{array}$

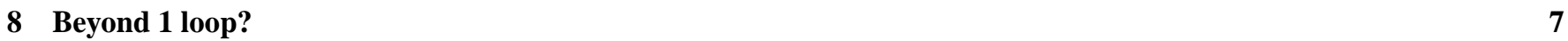

9 Results at 2-loop order $r$

$\begin{array}{lr}10 \text { Large } N & 8\end{array}$

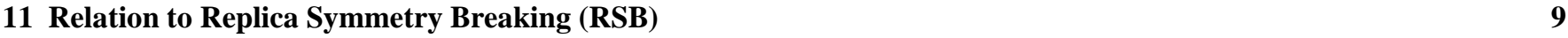

12 Finite $N \quad 11$

13 Depinning transition $r$

14 Universal width distribution $\quad 14$

15 Anisotropic depinning, directed percolation, branching and all that 15

16 Perspectives $\quad 17$ 


\section{Introduction}

Statistical mechanics is by now a rather mature branch of physics. For pure systems like a ferromagnet, it allows to calculate so precise details as the behavior of the specific heat on approaching the Curie-point. We know that it diverges as a function of the distance in temperature to the Curie-temperature, we know that this divergence has the form of a power-law, we can calculate the exponent, and we can do this with at least 3 digits of accuracy. Best of all, these findings are in excellent agreement with the most precise experiments. This is a true success story of statistical mechanics. On the other hand, in nature no system is really pure, i.e. without at least some disorder ("dirt"). As experiments (and theory) seem to suggest, a little bit of disorder does not change the behavior much. Otherwise experiments on the specific heat of Helium would not so extraordinarily well confirm theoretical predictions. But what happens for strong disorder? By this I mean that disorder completely dominates over entropy. Then already the question: "What is the ground-state?" is no longer simple. This goes hand in hand with the appearance of so-called metastable states. States, which in energy are very close to the ground-state, but which in configuration-space may be far apart. Any relaxational dynamics will take an enormous time to find the correct ground-state, and may fail altogether, as can be seen in computer-simulations as well as in experiments. This means that our way of thinking, taught in the treatment of pure systems, has to be adapted to account for disorder. We will see that in contrast to pure systems, whose universal large-scale properties can be described by very few parameters, disordered systems demand the knowledge of the whole disorder-distribution function (in contrast to its first few moments). We show how universality nevertheless emerges.

Experimental realizations of strongly disordered systems are glasses, or more specifically spin-glasses, vortexglasses, electron-glasses and structural glasses (not treated here). Furthermore random-field magnets, and last not least elastic systems in disorder.

What is our current understanding of disordered systems? It is here that the success story of statistical mechanics, with which I started, comes to an end: Despite 30 years of research, we do not know much: There are a few exact solutions, there are phenomenological methods (like the droplet-model), and there is the mean-field approximation, involving a method called replica-symmetry breaking (RSB). This method is correct for infinitely connected systems, e.g. the SK-model (Sherrington Kirkpatrick model), or for systems with infinitely many components. However it is unclear, to which extend it applies to real physical systems, in which each degree of freedom is only coupled to a finite number of other degrees of freedom.

Another interesting system are elastic manifolds in a random media, which has the advantage of being approachable by other (analytic) methods, while still retaining all the rich physics of strongly disordered systems. Here, I review recent advances obtained in collaboration with Pierre Le Doussal [1-12]. This review is an extended version of [13].

\section{Physical realizations, model and observables}

Before developing the theory to treat elastic systems in a disordered environment, let us give some physical realizations. The simplest one is an Ising magnet. Imposing boundary conditions with all spins up at the upper and all spins down at the lower boundary (see figure 1), at low temperatures, a domain wall separates a region with spin up from a region with spin down. In a pure system at temperature $T=0$, this domain wall is completely flat. Disorder can deform the domain wall, making it eventually rough again. Two types of disorder are common: random bond (which on a course-grained level also represents missing spins) and random field (coupling of the spins to an external random magnetic field). Figure 1 shows, how the domain wall is described by a displacement field $u(x)$. Another example is the contact line of water (or liquid Helium), wetting a rough substrate, see figure 2. (The elasticity is long range). A realization with a 2-parameter displacement field $\vec{u}(\vec{x})$ is the deformation of a vortex lattice: the position of each vortex is deformed from $\vec{x}$ to $\vec{x}+\vec{u}(\vec{x})$. A 3-dimensional example are charge density waves.

All these models have in common, that they are described by a displacement field

$$
x \in \mathbb{R}^{d} \longrightarrow \vec{u}(x) \in \mathbb{R}^{N}
$$



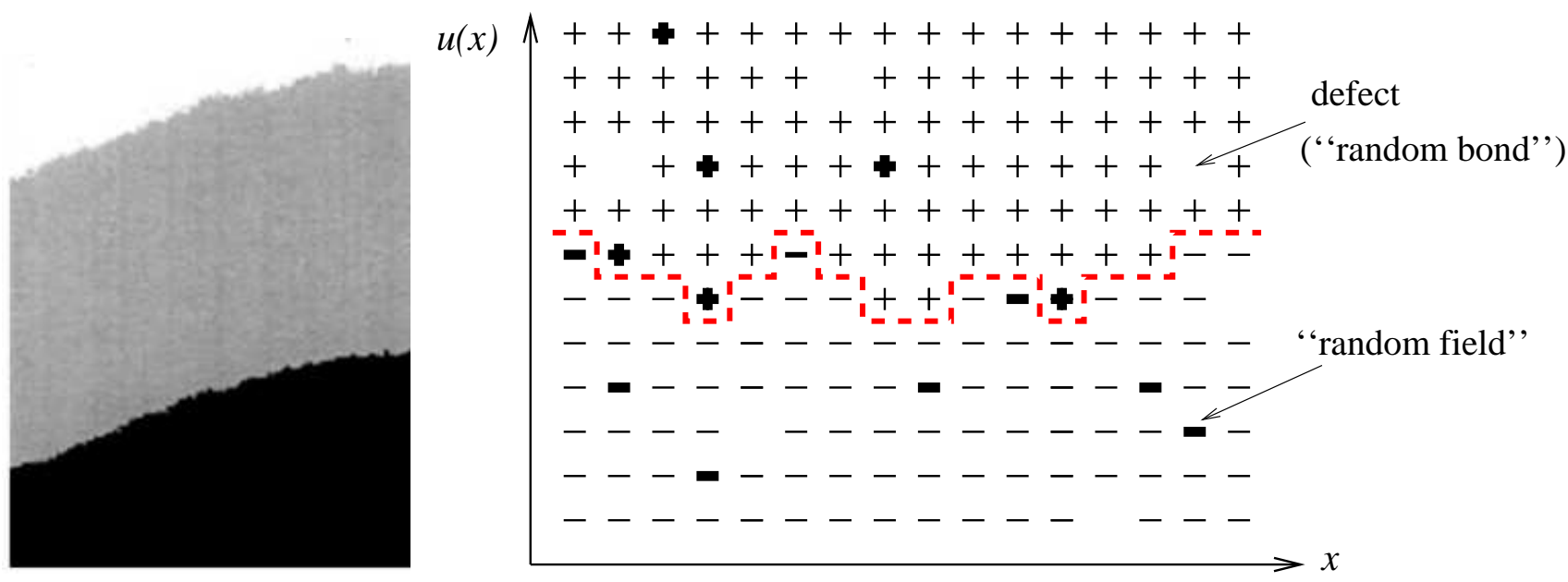

Figure 1: An Ising magnet at low temperatures forms a domain wall described by a function $u(x)$ (right). An experiment on a thin Cobalt film (left) [14]; with kind permission of the authors.

For simplicity, we set $N=1$ in the following. After some initial coarse-graining, the energy $\mathcal{H}=\mathcal{H}_{\mathrm{el}}+\mathcal{H}_{\mathrm{DO}}$ consists out of two parts: the elastic energy

$$
\mathcal{H}_{\mathrm{el}}[u]=\int \mathrm{d}^{d} x \frac{1}{2}(\nabla u(x))^{2}
$$

and the disorder

$$
\mathcal{H}_{\mathrm{DO}}[u]=\int \mathrm{d}^{d} x V(x, u(x)) .
$$

In order to proceed, we need to specify the correlations of disorder. Suppose that fluctuations $u$ in the transversal direction scale as

$$
\overline{(u(x)-u(y))^{2}} \sim|x-y|^{2 \zeta}
$$

with a roughness-exponent $\zeta<1$. Starting from a disorder correlator

$$
\overline{V(u, x) V\left(u^{\prime}, x^{\prime}\right)}=f\left(x-x^{\prime}\right) R\left(u-u^{\prime}\right)
$$
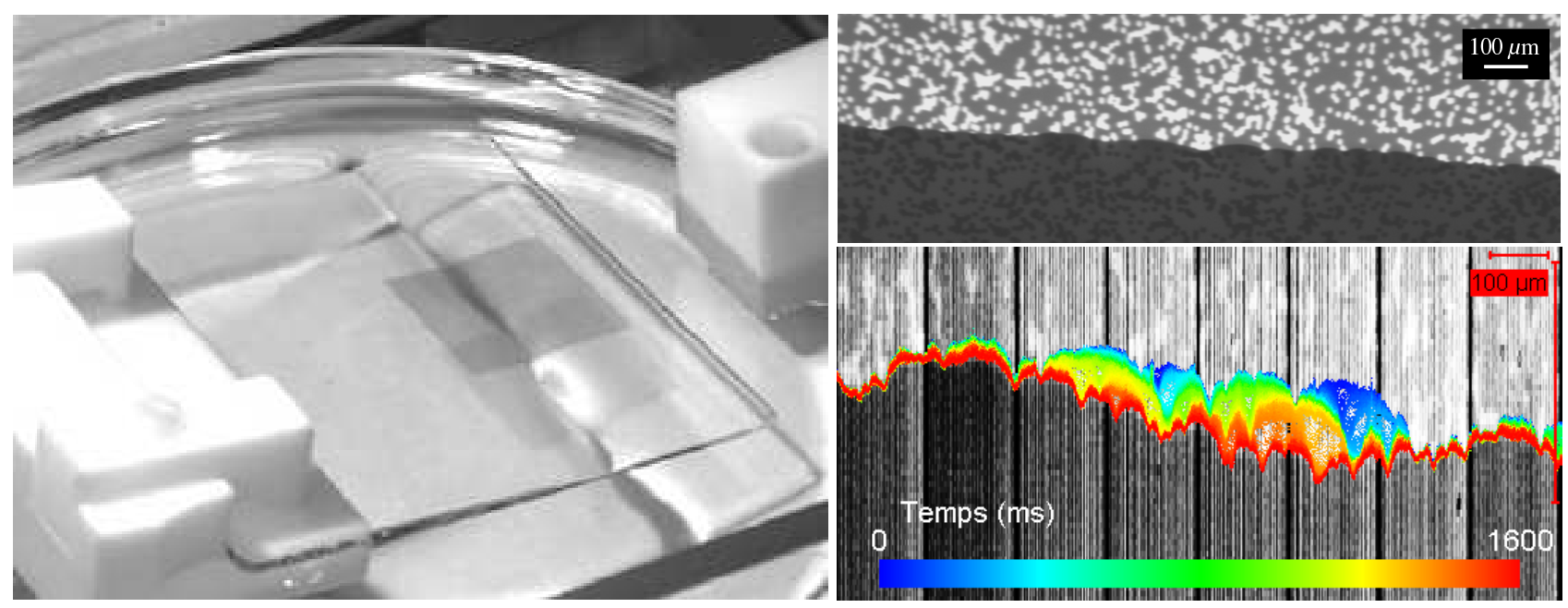

Figure 2: A contact line for the wetting of a disordered substrate by Glycerine [15]. Experimental setup (left). The disorder consists of randomly deposited islands of Chromium, appearing as bright spots (top right). Temporal evolution of the retreating contact-line (bottom right). Note the different scales parallel and perpendicular to the contact-line. Pictures courtesy of S. Moulinet, with kind permission. 


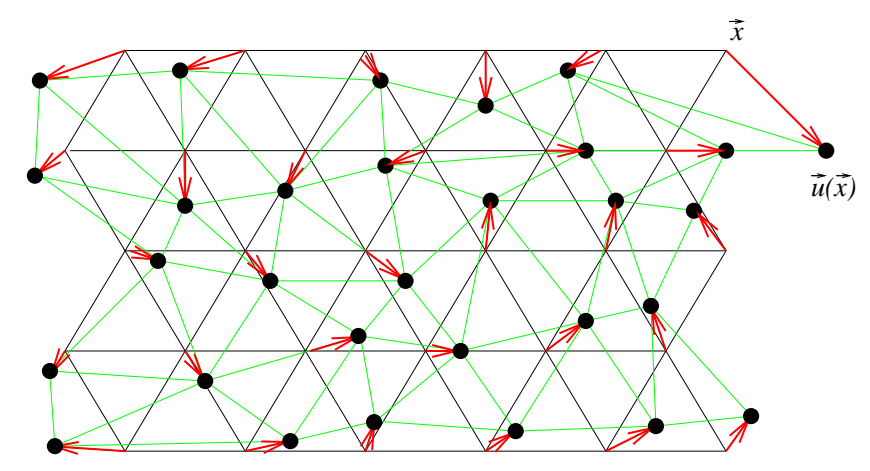

Figure 3: Cartoon of an elastic lattice (e.g. vortex lattice) deformed by disorder. This is described by a vector $\vec{u}(x)$.

and performing one step in the RG-procedure, one has to rescale more in the $x$-direction than in the $u$-direction. This will eventually reduce $f\left(x-x^{\prime}\right)$ to a $\delta$-distribution, whereas the structure of $R\left(u-u^{\prime}\right)$ remains visible. We therefore choose as our starting-model

$$
\overline{V(u, x) V\left(u^{\prime}, x^{\prime}\right)}:=\delta^{d}\left(x-x^{\prime}\right) R\left(u-u^{\prime}\right) .
$$

There are a couple of useful observables. We already mentioned the roughness-exponent $\zeta$. The second is the renormalized (effective) disorder. It will turn out that we actually have to keep the whole disorder distribution function $R(u)$, in contrast to keeping a few moments. Other observables are higher correlation functions or the free energy.

\section{Treatment of disorder}

Having defined our model, we can now turn to the treatment of disorder. The problem is to average not the partitionfunction, but the free energy over disorder: $\overline{\mathcal{F}}=\overline{\ln Z}$. This can be achieved by the beautiful replica-trick. The idea is to write

$$
\ln \mathcal{Z}=\lim _{n \rightarrow 0} \frac{1}{n}\left(\mathrm{e}^{n \ln \mathcal{Z}}-1\right)=\lim _{n \rightarrow 0} \frac{1}{n}\left(\mathcal{Z}^{n}-1\right)
$$

and to interpret $\mathcal{Z}^{n}$ as the partition-function of an $n$ times replicated system. Averaging $\mathrm{e}^{-\sum_{a=1}^{n} \mathcal{H}_{a}}$ over disorder then leads to the replica-Hamiltonian

$$
\mathcal{H}[u]=\frac{1}{T} \sum_{a=1}^{n} \int \mathrm{d}^{d} x \frac{1}{2}\left(\nabla u_{a}(x)\right)^{2}-\frac{1}{2 T^{2}} \sum_{a, b=1}^{n} \int \mathrm{d}^{d} x R\left(u_{a}(x)-u_{b}(x)\right) .
$$

Let us stress that one could equivalently pursue a dynamic or a supersymmetric formulation. We therefore should not, and in fact do not encounter, problems associated with the use of the replica-trick.

\section{Dimensional reduction}

There is a beautiful and rather mind-boggling theorem relating disordered systems to pure systems (i.e. without disorder), which applies to a large class of systems, e.g. random field systems and elastic manifolds in disorder. It is called dimensional reduction and reads as follows [16]:

Theorem: A d-dimensional disordered system at zero temperature is equivalent to all orders in perturbation theory to a pure system in $d-2$ dimensions at finite temperature. Moreover the temperature is (up to a constant) nothing but the width of the disorder distribution. A simple example is the 3-dimensional random-field Ising model at zero temperature; according to the theorem it should be equivalent to the pure 1-dimensional Ising-model at finite temperature. But it has been shown rigorously, that the former has an ordered phase, whereas we have all solved the latter and we know that there is no such phase at finite temperature. So what went wrong? Let me stress that there are no missing diagrams or any such thing, but that the problem is more fundamental: As we will see later, the proof makes 


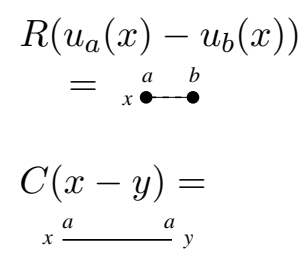

$$
\begin{aligned}
& \delta R\left(u_{a}-u_{b}\right)=\underbrace{}_{a-y} C(x-y)^{2}\left[R^{\prime \prime}\left(u_{a}-u_{b}\right)^{2}-2 R^{\prime \prime}\left(u_{a}-u_{b}\right) R^{\prime \prime}\left(u_{a}-u_{a}\right)\right] \\
& =\int_{a}^{b} b_{a}^{b}
\end{aligned}
$$

Figure 4: The disorder vertex $R\left(u_{a}(x)-u_{b}(x)\right)$ and the correlation-function $C(x-y)$, with Fourier-transform $\tilde{C}(k)=\frac{1}{k^{2}}$, which is diagonal in replica-space (left). Contracting two disorder-vertices with two correlation-functions leads to the two 1-loop contributions $\delta R$ to the disorder-correlator $R$ (right). The integral $\int_{x-y} C(x-y)^{2}=\frac{L^{\epsilon}}{\epsilon}$, where $L$ is some IR-cutoff.

assumptions, which are not satisfied. Nevertheless, the above theorem remains important since it has a devastating consequence for all perturbative calculations in the disorder: However clever a procedure we invent, as long as we do a perturbative expansion, expanding the disorder in its moments, all our efforts are futile: dimensional reduction tells us that we get a trivial and unphysical result. Before we try to understand why this is so and how to overcome it, let me give one more example. Dimensional reduction allows to calculate the roughness-exponent $\zeta$ defined in equation (2.4). We know (this can be inferred from power-counting) that the width $u$ of a $d$-dimensional manifold at finite temperature in the absence of disorder scales as $u \sim x^{(2-d) / 2}$. Making the dimensional shift implied by dimensional reduction leads to

$$
\overline{(u(x)-u(0))^{2}} \sim x^{4-d} \equiv x^{2 \zeta} \quad \text { i.e. } \quad \zeta=\frac{4-d}{2} .
$$

\section{The Larkin-length}

To understand the failure of dimensional reduction, let us turn to an interesting argument given by Larkin [17]. He considers a piece of an elastic manifold of size $L$. If the disorder has correlation length $r$, and characteristic potential energy $\bar{f}$, this piece will typically see a potential energy of strength

$$
E_{\mathrm{DO}}=\bar{f}\left(\frac{L}{r}\right)^{\frac{d}{2}} .
$$

On the other hand, there is an elastic energy, which scales like

$$
E_{\mathrm{el}}=c L^{d-2} .
$$

These energies are balanced at the Larkin-length $L=L_{c}$ with

$$
L_{c}=\left(\frac{c^{2}}{\bar{f}^{2}} r^{d}\right)^{\frac{1}{4-d}} .
$$

More important than this value is the observation that in all physically interesting dimensions $d<4$, and at scales $L>L_{c}$, the membrane is pinned by disorder; whereas on small scales elastic energy dominates. Since the disorder has a lot of minima which are far apart in configurational space but close in energy (metastability), the manifold can be in either of these minimas, and the ground-state is no longer unique. However exactly this is assumed in e.g. the proof of dimensional reduction; as is most easily seen in its supersymmetric formulation [18].

\section{The functional renormalization group (FRG)}

Let us now discuss a way out of the dilemma: Larkin's argument suggests that 4 is the upper critical dimension. So we would like to make an $\epsilon=4-d$ expansion. On the other hand, dimensional reduction tells us that the roughness is 

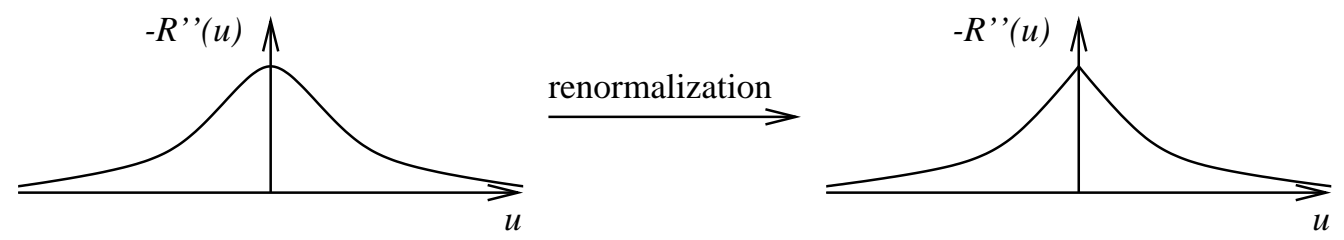

Figure 5: Change of $-R^{\prime \prime}(u)$ under renormalization and formation of the cusp.

$\zeta=\frac{4-d}{2}$ (see (4.1)). Even though this is systematically wrong below four dimensions, it tells us correctly that at the critical dimension $d=4$, where disorder is marginally relevant, the field $u$ is dimensionless. This means that having identified any relevant or marginal perturbation (as the disorder), we find immediately another such perturbation by adding more powers of the field. We can thus not restrict ourselves to keeping solely the first moments of the disorder, but have to keep the whole disorder-distribution function $R(u)$. Thus we need a functional renormalization group treatment (FRG). Functional renormalization is an old idea going back to the seventies, and can e.g. be found in [19]. For disordered systems, it was first proposed in 1986 by D. Fisher [20]. Performing an infinitesimal renormalization, i.e. integrating over a momentum shell à la Wilson, leads to the flow $\partial_{\ell} R(u)$, with $(\epsilon=4-d)$

$$
\partial_{\ell} R(u)=(\epsilon-4 \zeta) R(u)+\zeta u R^{\prime}(u)+\frac{1}{2} R^{\prime \prime}(u)^{2}-R^{\prime \prime}(u) R^{\prime \prime}(0) .
$$

The first two terms come from the rescaling of $R$ and $u$ respectively. The last two terms are the result of the 1-loop calculations, which are sketched in figure 4.

More important than the form of this equation is it actual solution, sketched in figure 5. After some finite renormalization, the second derivative of the disorder $R^{\prime \prime}(u)$ acquires a cusp at $u=0$; the length at which this happens is the Larkin-length. How does this overcome dimensional reduction? To understand this, it is interesting to study the flow of the second and forth moment. Taking derivatives of (6.1) w.r.t. $u$ and setting $u$ to 0 , we obtain

$$
\begin{aligned}
\partial_{\ell} R^{\prime \prime}(0) & =(\epsilon-2 \zeta) R^{\prime \prime}(0)+R^{\prime \prime \prime}(0)^{2} \longrightarrow(\epsilon-2 \zeta) R^{\prime \prime}(0) \\
\partial_{\ell} R^{\prime \prime \prime \prime}(0) & =\epsilon R^{\prime \prime \prime \prime}(0)+3 R^{\prime \prime \prime \prime}(0)^{2}+4 R^{\prime \prime \prime}(0) R^{\prime \prime \prime \prime \prime \prime}(0) \longrightarrow \epsilon R^{\prime \prime \prime \prime}(0)+3 R^{\prime \prime \prime \prime}(0)^{2} .
\end{aligned}
$$

Since $R(u)$ is an even function, $R^{\prime \prime \prime}(0)$ and $R^{\prime \prime \prime \prime \prime}(0)$ are 0 , which we have already indicated in Eqs. (6.2) and (6.3) . The above equations for $R^{\prime \prime}(0)$ and $R^{\prime \prime \prime \prime}(0)$ are in fact closed. Equation (6.2) tells us that the flow of $R^{\prime \prime}(0)$ is trivial and that $\zeta=\epsilon / 2 \equiv \frac{4-d}{2}$. This is exactly the result predicted by dimensional reduction. The appearance of the cusp can be inferred from equation (6.3). Its solution is

$$
\left.R^{\prime \prime \prime \prime}(0)\right|_{\ell}=\frac{c \mathrm{e}^{\epsilon \ell}}{1-3 c\left(\mathrm{e}^{\epsilon \ell}-1\right) / \epsilon}, \quad c:=\left.R^{\prime \prime \prime \prime}(0)\right|_{\ell=0}
$$

Thus after a finite renormalization $R^{\prime \prime \prime \prime}(0)$ becomes infinite: The cusp appears. By analyzing the solution of the flow-equation (6.1), one also finds that beyond the Larkin-length $R^{\prime \prime}(0)$ is no longer given by $(6.2)$ with $R^{\prime \prime \prime}(0)^{2}=0$. The correct interpretation of (6.2), which remains valid after the cusp-formation, is (for details see below)

$$
\partial_{\ell} R^{\prime \prime}(0)=(\epsilon-2 \zeta) R^{\prime \prime}(0)+R^{\prime \prime \prime}\left(0^{+}\right)^{2}
$$

Renormalization of the whole function thus overcomes dimensional reduction. The appearance of the cusp also explains why dimensional reduction breaks down. The simplest way to see this is by redoing the proof for elastic manifolds in disorder, which in the absence of disorder is a simple Gaussian theory. Terms contributing to the 2-point function involve $R^{\prime \prime}(0), T R^{\prime \prime \prime \prime}(0)$ and higher derivatives of $R(u)$ at $u=0$, which all come with higher powers of $T$. To obtain the limit of $T \rightarrow 0$, one sets $T=0$, and only $R^{\prime \prime}(0)$ remains. This is the dimensional reduction result. However we just saw that $R^{\prime \prime \prime \prime}(0)$ becomes infinite. Thus $R^{\prime \prime \prime \prime}(0) T$ may also contribute, and the proof fails. 


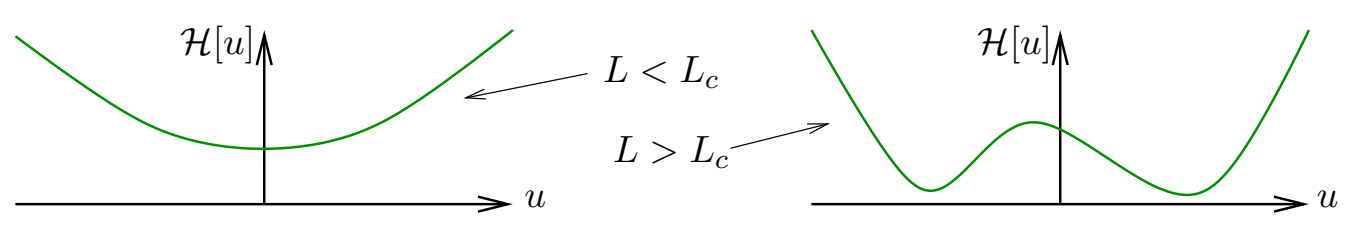

Figure 6: The toy model (7.1) before (left) and after (right) the Larkin-scale.

\section{Why is a cusp necessary?}

The appearance of a cusp might suggest that this approach is fatally ill. Let me present a simple argument due to Leon Balents [21], why a cusp is a physical necessity and not an artifact. To this aim, consider a toy model with only one Fourier-mode $u=u_{q}$

$$
\mathcal{H}[u]=\frac{1}{2} q^{2} u^{2}+\sqrt{\epsilon} \tilde{V}(u) .
$$

Since equation (6.1) has a fixed point of order $R(u) \sim \epsilon$ for all $\epsilon>0, V(u)$ scales like $\sqrt{\epsilon}$ for $\epsilon$ small and we have made this dependence explicit in (7.1) by using $V(u)=\sqrt{\epsilon} \tilde{V}(u)$. The only further input comes from the physics: For $L<L_{c}$, i.e. before we reach the Larkin length, there is only one minimum, as depicted in figure 6 . On the other hand, for $L>L_{c}$, there are several minima. Thus there is at least one point for which

$$
\frac{\mathrm{d}^{2}}{\mathrm{~d} u^{2}} \mathcal{H}[u]=q^{2}+\sqrt{\epsilon} \tilde{V}^{\prime \prime}(u)<0 .
$$

In the limit of $\epsilon \rightarrow 0$, this is possible if and only if $\frac{1}{\epsilon} R^{\prime \prime \prime \prime}(0)$, which a priori should be finite for $\epsilon \rightarrow 0$, becomes infinite:

$$
\frac{1}{\epsilon} R^{\prime \prime \prime \prime}(0)=\left.\overline{V^{\prime \prime}(u) V^{\prime \prime}\left(u^{\prime}\right)}\right|_{u=u^{\prime}}=\infty
$$

This argument shows that a cusp is indeed a physical necessity.

\section{Beyond 1 loop?}

Functional renormalization has successfully been applied to a bunch of problems at 1-loop order. From a field theory, we however demand more. Namely that it

- allows for systematic corrections beyond 1-loop order

- be renormalizable

- and thus allows to make universal predictions.

However, this has been a puzzle since 1986, and it has even been suggested that the theory is not renormalizable due to the appearance of terms of order $\epsilon^{\frac{3}{2}}$ [22]. Why is the next order so complicated? The reason is that it involves terms proportional to $R^{\prime \prime \prime}(0)$. A look at figure 3 explains the puzzle. Shall we use the symmetry of $R(u)$ to conclude that $R^{\prime \prime \prime}(0)$ is 0 ? Or shall we take the left-hand or right-hand derivatives, related by

$$
R^{\prime \prime \prime}\left(0^{+}\right):=\lim _{\substack{u>0 \\ u \rightarrow 0}} R^{\prime \prime \prime}(u)=-\lim _{\substack{u<0 \\ u \rightarrow 0}} R^{\prime \prime \prime}(u)=:-R^{\prime \prime \prime}\left(0^{-}\right) .
$$

In the following, I will present our solution of this puzzle, obtained at 2-loop order in [1] and then at large $N$ in [2]. The latter approach allows for another independent control-parameter, and sheds further light on the cusp-formation. We refer the reader to these works for further references. 


\begin{tabular}{|c|c|c|c|c|}
\hline$\zeta$ & one loop & two loop & estimate & simulation and exact \\
\hline \hline$d=3$ & 0.208 & 0.215 & $0.215 \pm 0.01$ & $0.22 \pm 0.01[23]$ \\
\hline$d=2$ & 0.417 & 0.444 & $0.42 \pm 0.02$ & $0.41 \pm 0.01[23]$ \\
\hline$d=1$ & 0.625 & 0.687 & $0.67 \pm 0.02$ & $2 / 3$ \\
\hline
\end{tabular}

Figure 7: Results for $\zeta$ in the random bond case.

\section{Results at 2-loop order}

For the flow-equation at 2-loop order, we find [1]

$$
\begin{aligned}
\partial_{\ell} R(u)= & (\epsilon-4 \zeta) R(u)+\zeta u R^{\prime}(u)+\frac{1}{2} R^{\prime \prime}(u)^{2}-R^{\prime \prime}(u) R^{\prime \prime}(0) \\
& +\frac{1}{2}\left(R^{\prime \prime}(u)-R^{\prime \prime}(0)\right) R^{\prime \prime \prime}(u)^{2}-\frac{1}{2} R^{\prime \prime \prime}\left(0^{+}\right)^{2} R^{\prime \prime}(u) .
\end{aligned}
$$

The first line is the result at 1-loop order, already given in (6.1). The second line is new. The most interesting term is the last one, which involves $R^{\prime \prime \prime}\left(0^{+}\right)^{2}$ and which we therefore call anomalous. The hard task is to fix the prefactor $\left(-\frac{1}{2}\right)$. We have up to now invented six algorithms to do so; one leads to inconsistencies and shall not be reported here. The other five algorithms are consistent with each other: The sloop-algorithm, recursive construction, reparametrization invariance, renormalizability, and potentiality [1,4]. For lack of space, we restrain our discussion to the last two ones. At 2-loop order the following diagram appears

$$
R_{R^{\prime \prime}} \rightarrow \frac{1}{2}\left(R^{\prime \prime}(u)-R^{\prime \prime}(0)\right) R^{\prime \prime \prime}(u)^{2}-\frac{1}{2} R^{\prime \prime}(u) R^{\prime \prime \prime}\left(0^{+}\right)^{2}
$$

leading to the anomalous term. The integral (not written here) contains a subdivergence, which is indicated by the box. Renormalizability demands that its leading divergence (which is of order $1 / \epsilon^{2}$ ) be canceled by a 1-loop counterterm. The latter is unique thus fixing the prefactor of the anomalous term. (The idea is to take the 1-loop correction $\delta R$ in figure 2 and replace one of the $R^{\prime \prime}$ in it by $\delta R^{\prime \prime}$ itself, which the reader can check to leading to the terms given in (9.2) plus terms which only involve even derivatives.)

Another very physical demand is that the problem remain potential, i.e. that forces still derive from a potential. The force-force correlation function being $-R^{\prime \prime}(u)$, this means that the flow of $R^{\prime}(0)$ has to be strictly 0 . (The simplest way to see this is to study a periodic potential.) From (9) one can check that this does not remain true if one changes the prefactor of the last term in (9); thus fixing it.

Let us give some results for cases of physical interest. First of all, in the case of a periodic potential, which is relevant for charge-density waves, the fixed-point function can be calculated analytically as (we choose period 1, the following is for $u \in[0,1]$ )

$$
R^{*}(u)=-\left(\frac{\epsilon}{72}+\frac{\epsilon^{2}}{108}+O\left(\epsilon^{3}\right)\right) u^{2}(1-u)^{2}+\text { const. }
$$

This leads to a universal amplitude. In the case of random field disorder (short-ranged force-force correlation function) $\zeta=\frac{\epsilon}{3}$. For random-bond disorder (short-ranged potential-potential correlation function) we have to solve (9.1) numerically, with the result $\zeta=0.20829804 \epsilon+0.006858 \epsilon^{2}$. This compares well with numerical simulations, see figure 7.

\section{Large $N$}

In the last section, we have discussed renormalization in a loop expansion, i.e. expansion in $\epsilon$. In order to independently check consistency it is good to have a non-perturbative approach. This is achieved by the large- $N$ limit, which 
can be solved analytically and to which we turn now. We start from

$$
\begin{aligned}
\mathcal{H}[\vec{u}, \vec{j}]= & \frac{1}{2 T} \sum_{a=1}^{n} \int_{x} \vec{u}_{a}(x)\left(-\nabla^{2}+m^{2}\right) \vec{u}_{a}(x)-\sum_{a=1}^{n} \int_{x} \vec{j}_{a}(x) \vec{u}_{a}(x) \\
& -\frac{1}{2 T^{2}} \sum_{a, b=1}^{n} \int_{x} B\left(\left(\vec{u}_{a}(x)-\vec{u}_{b}(x)\right)^{2}\right) .
\end{aligned}
$$

where in contrast to (3.2), we use an $N$-component field $\vec{u}$. For $N=1$, we identify $B\left(u^{2}\right)=R(u)$. We also have added a mass $m$ to regularize the theory in the infra-red and a source $\vec{j}$ to calculate the effective action $\Gamma(\vec{u})$ via a Legendre transform. For large $N$ the saddle point equation reads [2]

$$
\tilde{B}^{\prime}\left(u_{a b}^{2}\right)=B^{\prime}\left(u_{a b}^{2}+2 T I_{1}+4 I_{2}\left[\tilde{B}^{\prime}\left(u_{a b}^{2}\right)-\tilde{B}^{\prime}(0)\right]\right)
$$

This equation gives the derivative of the effective (renormalized) disorder $\tilde{B}$ as a function of the (constant) background field $u_{a b}^{2}=\left(u_{a}-u_{b}\right)^{2}$ in terms of: the derivative of the microscopic (bare) disorder $B$, the temperature $T$ and the integrals $I_{n}:=\int_{k} \frac{1}{\left(k^{2}+m^{2}\right)^{n}}$.

The saddle-point equation can again be turned into a closed functional renormalization group equation for $\tilde{B}$ by taking the derivative w.r.t. $m$ :

$$
\partial_{l} \tilde{B}(x) \equiv-\frac{m \partial}{\partial m} \tilde{B}(x)=(\epsilon-4 \zeta) \tilde{B}(x)+2 \zeta x \tilde{B}^{\prime}(x)+\frac{1}{2} \tilde{B}^{\prime}(x)^{2}-\tilde{B}^{\prime}(x) \tilde{B}^{\prime}(0)+\frac{\epsilon T \tilde{B}^{\prime}(x)}{\epsilon+\tilde{B}^{\prime \prime}(0)}
$$

This is a complicated nonlinear partial differential equation. It is therefore surprising, that one can find an analytic solution. (The trick is to write down the flow-equation for the inverse function of $\tilde{B}^{\prime}(x)$, which is linear.) Let us only give the results of this analytic solution: First of all, for long-range correlated disorder of the form $\tilde{B}^{\prime}(x) \sim x^{-\gamma}$, the exponent $\zeta$ can be calculated analytically as $\zeta=\frac{\epsilon}{2(1+\gamma)}$. It agrees with the replica-treatment in [24] and the 1-loop treatment in [22]. For short-range correlated disorder, $\zeta=0$. Second, it demonstrates that before the Larkin-length, $\tilde{B}(x)$ is analytic and thus dimensional reduction holds. Beyond the Larkin length, $\tilde{B}^{\prime \prime}(0)=\infty$, a cusp appears and dimensional reduction is incorrect. This shows again that the cusp is not an artifact of the perturbative expansion, but an important property even of the exact solution of the problem (here in the limit of large $N$ ).

\section{Relation to Replica Symmetry Breaking (RSB)}

There is another treatment of the limit of large $N$ given by Mézard and Parisi [24]. They start from (10.1) but without a source-term $j$. In the limit of large $N$, a Gaussian variational ansatz of the form

$$
\mathcal{H}_{\mathrm{g}}[\vec{u}]=\frac{1}{2 T} \sum_{a=1}^{n} \int_{x} \vec{u}_{a}(x)\left(-\nabla^{2}+m^{2}\right) \vec{u}_{a}(x)-\frac{1}{2 T^{2}} \sum_{a, b=1}^{n} \sigma_{a b} \vec{u}_{a}(x) \vec{u}_{b}(x)
$$

becomes exact. The art is to make an appropriate ansatz for $\sigma_{a b}$. The simplest possibility, $\sigma_{a b}=\sigma$ for all $a \neq b$ reproduces the dimensional reduction result, which breaks down at the Larkin length. Beyond that scale, a replica symmetry broken (RSB) ansatz for $\sigma_{a b}$ is suggestive. To this aim, one can break $\sigma_{a b}$ into four blocks of equal size, choose one (variationally optimized) value for the both outer diagonal blocks, and then iterate the procedure on the 
diagonal blocks, resulting in

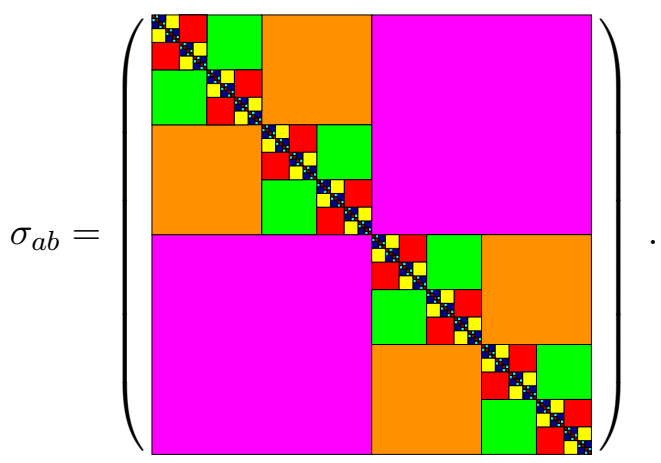

One finds that the more often one iterates, the better the results. In fact, one has to repeat this procedure infinite many times. This seems like a hopeless endeavor, but Parisi has shown that the infinitely often replica symmetry broken matrix can be parameterized by a function $[\sigma](z)$ with $z \in[0,1]$. In the SK-model, $z$ has the interpretation of an overlap between replicas. While there is no such simple interpretation for the model (11.1), we retain that $z=0$ describes distant states, whereas $z=1$ describes nearby states. The solution of the large- $N$ saddle-point equations leads to the curve depicted in figure 6. Knowing it, the 2-point function is given by

$$
\left\langle u_{k} u_{-k}\right\rangle=\frac{1}{k^{2}}\left(1+\int_{0}^{1} \frac{\mathrm{d} z}{z^{2}} \frac{[\sigma](z)+m^{2}}{k^{2}+[\sigma](z)+m^{2}}\right) .
$$

The important question is: What is the relation between the two approaches, which both pretend to calculate the same 2-point function? Comparing the analytical solutions, we find that the 2-point function given by FRG is the same as that of RSB, if in the latter expression we only take into account the contribution from the most distant states, i.e. those for $z$ between 0 and $z_{m}$ (see figure 8). To understand why this is so, we have to remember that the two calculations were done under quite different assumptions: In contrast to the RSB-calculation, the FRG-approach calculated the partition function in presence of an external field $j$, which was then used to give via a Legendre transformation the effective action. Even if the field $j$ is finally turned to 0 , the system might remember its preparation, as is the case for a magnet: Preparing the system in presence of a magnetic field will result in a magnetization which aligns with this field. The magnetization will remain, even if finally the field is turned off. The same phenomena happens here: By explicitly breaking the replica-symmetry through an applied field, all replicas will settle in distant states, and the close states from the Parisi-function $[\sigma](z)+m^{2}$ (which describes spontaneous RSB) will not contribute. However, we found that the full RSB-result can be reconstructed by remarking that the part of the curve between $z_{m}$ and $z_{c}$ is independent of the infrared cutoff $m$, and then integrating over $m$ [2] $\left(m_{c}\right.$ is the mass corresponding to $\left.z_{c}\right)$ :

$$
\left.\left\langle u_{k} u_{-k}\right\rangle\right|_{k=0} ^{\mathrm{RSB}}=\frac{\tilde{R}_{m}^{\prime}(0)}{m^{4}}+\int_{m}^{m_{c}} \frac{\mathrm{d} \tilde{R}_{\mu}^{\prime}(0)}{\mu^{4}}+\frac{1}{m_{c}^{2}}-\frac{1}{m^{2}} .
$$

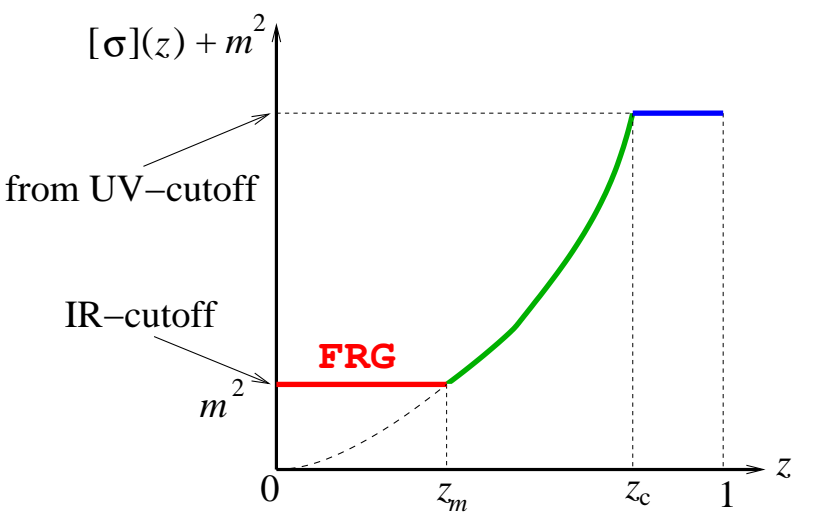

Figure 8: The function $[\sigma](u)+m^{2}$ as given in [24]. 
We also note that a similar effective action has been proposed in [25]. While it agrees qualitatively, it does not reproduce the correct FRG 2-point function, as it should.

\section{Finite $N$}

Up to now, we have studied the functional RG in two cases: For one component $N=1$ and in the limit of a large number of components, $N \rightarrow \infty$. The general case of finite $N$ is more difficult to handle, since derivatives of the renormalized disorder now depend on the direction, in which this derivative are taken. Define amplitude $u:=|\vec{u}|$ and direction $\hat{u}:=\vec{u} /|\vec{u}|$ of the field. Then deriving the latter variable leads to terms proportional to $1 / u$, which are diverging in the limit of $u \rightarrow 0$. This poses additional problems in the calculation, and it is a priori not clear that the theory at $N \neq 1$ exists, supposed this is the case for $N=1$. At 1-loop order everything is well-defined [22]. We have found a consistent RG-equation at 2-loop order [8]:

$$
\begin{aligned}
\partial_{\ell} R(u)= & (\epsilon-4 \zeta) R(u)+\zeta u R^{\prime}(u)+\frac{1}{2} R^{\prime \prime}(u)^{2}-R^{\prime \prime}(0) R^{\prime \prime}(u)+\frac{N-1}{2} \frac{R^{\prime}(u)}{u}\left(\frac{R^{\prime}(u)}{u}-2 R^{\prime \prime}(0)\right) \\
& +\frac{1}{2}\left(R^{\prime \prime}(u)-R^{\prime \prime}(0)\right) R^{\prime \prime \prime}(u)^{2}+\frac{N-1}{2} \frac{\left(R^{\prime}(u)-u R^{\prime \prime}(u)\right)^{2}\left(2 R^{\prime}(u)+u\left(R^{\prime \prime}(u)-3 R^{\prime \prime}(0)\right)\right)}{u^{5}} \\
& -R^{\prime \prime \prime}\left(0^{+}\right)^{2}\left[\frac{N+3}{8} R^{\prime \prime}(u)+\frac{N-1}{4} \frac{R^{\prime}(r)}{u}\right] .
\end{aligned}
$$

The first line is the 1-loop equation, given in [22]. The second and third line represent the 2-loop equation, with the new anomalous terms proportional to $R^{\prime \prime \prime}\left(0^{+}\right)^{2}$ (third line).

The fixed point equation (12.1) can be integrated numerically, order by order in $\epsilon$. The result, specialized to directed polymers, i.e. $\epsilon=3$ is plotted on figure 9. We see that the 2-loop corrections are rather big at large $N$, so some doubt on the applicability of the latter down to $\epsilon=3$ is advised. However both 1- and 2-loop results reproduce well the two known points on the curve: $\zeta=2 / 3$ for $N=1$ and $\zeta=0$ for $N=\infty$. The latter result has been given in section 10. Via the equivalence [26] of the directed polymer problem in $N$ dimensions treated here and the KPZ-equation of non-linear surface growth in $N$ dimensions, which relate the roughness exponent $\zeta$ of the directed polymer to the dynamic exponent $z_{\mathrm{KPZ}}$ in the KPZ-equation via $\zeta=\frac{1}{z_{\mathrm{KPZ}}}$, we know that $\zeta(N=1)=2 / 3$.

The line $\zeta=1 / 2$ given on figure 9 plays a special role: In the presence of thermal fluctuations, we expect the roughness-exponent of the directed polymer to be bounded by $\zeta \geq 1 / 2$. In the KPZ-equation, this corresponds to a dynamic exponent $z_{\mathrm{KPZ}}=2$, which via the exact scaling relation $z_{\mathrm{KPZ}}+\zeta_{\mathrm{KPZ}}=2$ is an upper bound in the strong-coupling phase. The above data thus strongly suggest that there exists an upper critical dimension in the KPZproblem, with $d_{\mathrm{uc}} \approx 2.4$. Even though the latter value might be an underestimation, it is hard to imagine what can go wrong qualitatively with this scenario. The strongest objections will probably arize from numerical simulations,

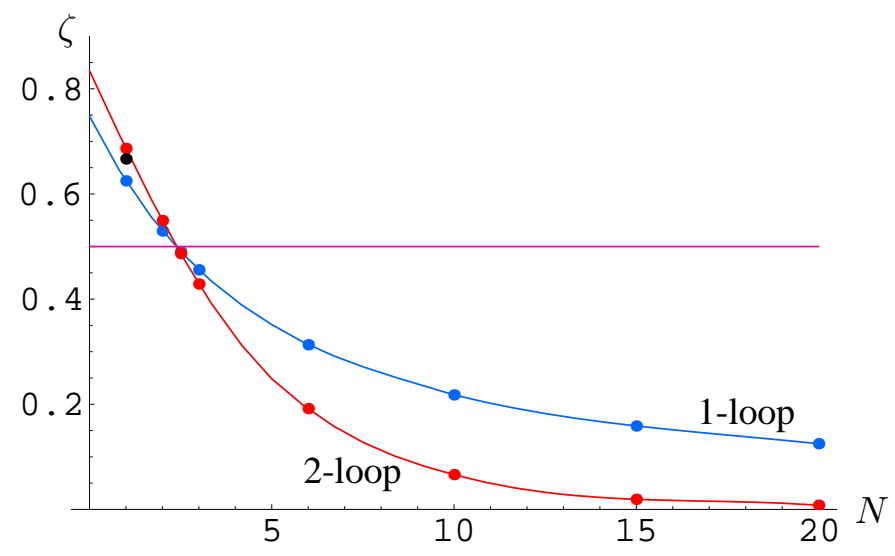

Figure 9: Results for the roughness $\zeta$ at 1- and 2-loop order, as a function of the number of components $N$. 
such as [27]. However the latter use a discrete RSOS model, and the exponents are measured for interfaces, which in large dimensions have the thickness of the discretization size, suggestions that the data are far from the asymptotic regime. We thus strongly incourage better numerical simulations on a continuous model, in order to settle this issue.

\section{Depinning transition}

Another important class of phenomena for elastic manifolds in disorder is the so-called "depinning transition": Applying a constant force to the elastic manifold, e.g. a constant magnetic field to the ferromagnet mentioned in the introduction, the latter will only move, if a certain critical threshhold force $f_{c}$ is surpassed, see figure 10. (This is fortunate, since otherwise the magnetic domain walls in the hard-disc drive onto which this article is stored would move with the effect of deleting all information, depriving the reader from his reading.) At $f=f_{c}$, the so-called depinning transition, the manifold has a distinctly different roughness exponent $\zeta$ (see Eq. (2.4)) from the equilibrium $(f=0)$. For $f>f_{c}$, the manifold moves, and close to the transition, new observables and corresponding exponents appear:

- the dynamic exponent $z$ relating correlation functions in spatial and temporal direction

$$
t \sim x^{z}
$$

- a correlation length $\xi$ set by the distance to $f_{c}$

$$
\xi \sim\left|f-f_{c}\right|^{-\nu}
$$

- furthermore, the new exponents are not all independent, but satisfy the following exponent relations [28]

$$
\beta=\nu(z-\zeta) \quad \nu=\frac{1}{2-\zeta}
$$

The equation describing the movement of the interface is

$$
\partial_{t} u(x, t)=\left(\nabla^{2}+m^{2}\right) u(x, t)+F(u(x, t), x), \quad F(u, x)=-\partial_{u} V(u, x)
$$

This model has been treated at 1-loop order by Natterman et al. [28] and by Narayan and Fisher [29]. The 1-loop flow-equations are identical to those of the statics. This is surprising, since physically, the phenomena at equilibrium and at depinning are quite different. There is even the claim by [29], that the roughness exponent in the random field universality class is exactly $\zeta=\epsilon / 3$, as it is in the equilibrium random field class. After a long debate among numerical physicists, the issue is today resolved: The roughness is significantly larger, and reads e.g. for the driven polymer $\zeta=1.25$, instead of $\zeta=1$ as predicted in [29]. Clearly, a 2-loop analysis [3] is necessary, to resolve these issues. Such a treatment starts from the dynamic action

$$
\mathcal{S}=\int_{x, t} \tilde{u}(x, t)\left(\partial_{t}-\nabla^{2}+m^{2}\right) u(x, t)+\int_{x, t, t^{\prime}} \tilde{u}(x, t) \Delta\left(u(x, t)-u\left(x, t^{\prime}\right)\right) \tilde{u}\left(x, t^{\prime}\right),
$$

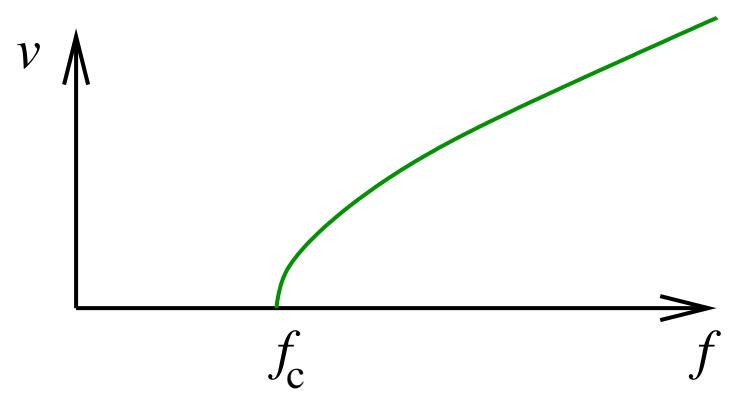

Figure 10: Velocity of a pinned interface as a function of the applied force. Zero force: equilibrium. $f=f_{c}$ : depinning. 
where the "response field" $\tilde{u}(x, t)$ enforces the equation of motion (13.2) and

$$
\overline{F(u, x) F\left(u^{\prime}, x^{\prime}\right)}=\Delta\left(u-u^{\prime}\right) \delta^{d}\left(x-x^{\prime}\right) \equiv-R^{\prime \prime}\left(u-u^{\prime}\right) \delta^{d}\left(x-x^{\prime}\right)
$$

is the force-force correlator, leading to the second term in (13.3). As in the statics, one encounters terms proportional to $\Delta^{\prime}\left(0^{+}\right) \equiv-R^{\prime \prime \prime}\left(0^{+}\right)$. Here the sign-problem can uniquely be resolved by supposing that the membrane only jumps ahead,

$$
t>t^{\prime} \quad \Rightarrow \quad u(x, t) \geq u\left(x, t^{\prime}\right) .
$$

Practically this means that when evaluating diagrams containing $\Delta\left(u(x, t)-u\left(x, t^{\prime}\right)\right)$, one splits them into two pieces, one with $t<t^{\prime}$ and one with $t>t^{\prime}$. Both pieces are well defined, even in the limit of $t \rightarrow t^{\prime}$. As the only tread-off of this method, diagrams can become complicated and difficult to evaluate; however they are always well-defined.

Physically, this means that we approach the depinning transition from above. This is reflected in (13.5) by the fact that $u(x, t)$ may remain constant; and indeed correlation-functions at the depinning transition are completely independent of time [3]. On the other hand a theory for the approach of the depinning transition from below $\left(f<f_{c}\right)$ has been elusive so far.

At the depinning transition, the 2-loop functional RG reads $[1,3]$

$$
\begin{aligned}
\partial_{\ell} R(u)= & (\epsilon-4 \zeta) R(u)+\zeta u R^{\prime}(u)+\frac{1}{2} R^{\prime \prime}(u)^{2}-R^{\prime \prime}(u) R^{\prime \prime}(0) \\
& +\frac{1}{2}\left[R^{\prime \prime}(u)-R^{\prime \prime}(0)\right] R^{\prime \prime \prime}(u)^{2}+\frac{1}{2} R^{\prime \prime \prime}\left(0^{+}\right)^{2} R^{\prime \prime}(u)
\end{aligned}
$$

First of all, note that it is a priori not clear that the functional RG equation, which is a flow equation for $\Delta(u)=$ $-R^{\prime \prime}(u)$ can be integrated to a functional RG-equation for $R(u)$. We have chosen this representation here, in order to make the difference to the statics evident: The only change is in the last sign on the second line of (13.6). This has important consequences for the physics: First of all, the roughness exponent $\zeta$ for the random-field universality class changes from $\zeta=\frac{\epsilon}{3}$ to

$$
\zeta=\frac{\epsilon}{3}(1+0.14331 \epsilon+\ldots)
$$

Second, the random-bond universality class is unstable and always renormalizes to the random-field universality class, as is physically expected: Since the membrane only jumps ahead, it always experiences a new disorder configuration, and there is no way to know of wether this disorder can be derived from a potential or not. This non-potentiality is most strikingly observed in the random periodic universality class, which is the relevannt one for charge density waves. The fixed point for a periodic disorder of period one reads (remember $\Delta(u)=-R^{\prime \prime}(u)$ )

$$
\Delta^{*}(u)=\frac{\epsilon}{36}+\frac{\epsilon^{2}}{108}-\left(\frac{\epsilon}{6}+\frac{\epsilon^{2}}{9}\right) u(1-u)
$$

\begin{tabular}{|r|c|c|c|c|r|}
\hline & $d$ & $\epsilon$ & $\epsilon^{2}$ & estimate & simulation \\
\hline \hline & 3 & 0.33 & 0.38 & $0.38 \pm 0.02$ & $0.34 \pm 0.01$ \\
\hline$\zeta$ & 2 & 0.67 & 0.86 & $0.82 \pm 0.1$ & $0.75 \pm 0.02$ \\
\hline & 1 & 1.00 & 1.43 & $1.2 \pm 0.2$ & $1.25 \pm 0.01$ \\
\hline \hline & 3 & 0.89 & 0.85 & $0.84 \pm 0.01$ & $0.84 \pm 0.02$ \\
\hline$\beta$ & 2 & 0.78 & 0.62 & $0.53 \pm 0.15$ & $0.64 \pm 0.02$ \\
\hline & 1 & 0.67 & 0.31 & $0.2 \pm 0.2$ & $0.25 \ldots 0.4$ \\
\hline \hline & 3 & 0.58 & 0.61 & $0.62 \pm 0.01$ & \\
\hline$\nu$ & 2 & 0.67 & 0.77 & $0.85 \pm 0.1$ & $0.77 \pm 0.04$ \\
\hline & 1 & 0.75 & 0.98 & $1.25 \pm 0.3$ & $1 \pm 0.05$ \\
\hline
\end{tabular}

\begin{tabular}{|c|c|c|c|c|}
\hline & $\epsilon$ & $\epsilon^{2}$ & estimate & simulation \\
\hline \hline$\zeta$ & 0.33 & 0.47 & $0.47 \pm 0.1$ & $0.39 \pm 0.002$ \\
\hline$\beta$ & 0.78 & 0.59 & $0.6 \pm 0.2$ & $0.68 \pm 0.06$ \\
\hline$z$ & 0.78 & 0.66 & $0.7 \pm 0.1$ & $0.74 \pm 0.03$ \\
\hline$\nu$ & 1.33 & 1.58 & $2 \pm 0.4$ & $1.52 \pm 0.02$ \\
\hline
\end{tabular}

Figure 11: The critical exponents at the depinning transition, for short range elasticity (left) and for long range elasticity (right). 
Integrating over a period, we find (suppressing in $F(u, x)$ the dependence on the coordinate $x$ for simplicity of notation)

$$
\int_{0}^{1} \mathrm{~d} u \Delta^{*}(u) \equiv \int_{0}^{1} \mathrm{~d} u \overline{F(u) F\left(u^{\prime}\right)}=-\frac{\epsilon^{2}}{108} .
$$

In an equilibrium situation, this correlator would vanish, since potentiality requires $\int_{0}^{1} \mathrm{~d} u F(u) \equiv 0$. Here, there are non-trivial contributions at 2-loop order (order $\epsilon^{2}$ ), violating this condition and rendering the problem non-potential. This same mechanism is also responsible for the violation of the conjecture $\zeta=\frac{\epsilon}{3}$, which could be proven on the assumption that the problem remains potential under renormalization. Let us stress that the breaking of potentiality under renormalization is a quite novel observation here.

The other critical exponents mentioned above can also be calculated. The dynamical exponent $z$ (for RF-disorder) reads $[1,3]$

$$
z=2-\frac{2}{9} \epsilon-0.04321 \epsilon^{2}+\ldots
$$

All other exponents are related via the relation (13.1). That the method works well even quantitatively can be infered from figure 11 .

\section{Universal width distribution}

Exponents are not the only interesting quantities: In experiments and simulations, often whole distributions can be measured, as e.g. the universal width distribution of an interface that we have computed at depinning [12,11]. Be $\langle u\rangle$ the average position of an interface for a given disorder configuration, then the spatially averaged width

$$
w^{2}:=\frac{1}{L^{d}} \int_{x}(u(x)-\langle u\rangle)^{2}
$$

is a random variable, and we can try to calculate and measure its distribution $P\left(w^{2}\right)$. The rescaled function $\Phi(z)$, defined by

$$
P\left(w^{2}\right)=1 / \overline{w^{2}} \Phi\left(w^{2} / \overline{w^{2}}\right)
$$

will be universal, i.e. independent of microscopic details and the size of the system.

Supposing all correlations to be Gaussian, $\Phi(z)$ can be calculated analytically. It depends on two parameters, the roughness exponent $\zeta$ and the dimension $d$. Numerical simulations displayed on figure 12 show spectacular

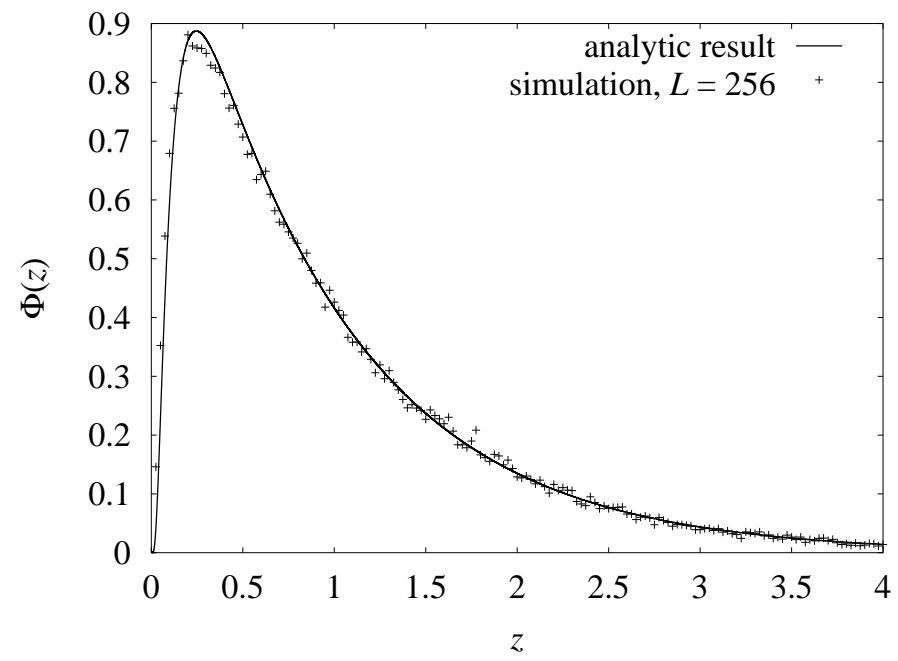

Figure 12: Scaling function $\Phi(z)$ for the $(1+1)$-dimensional harmonic model, compared to the Gaussian approximation for $\zeta=1.25$. Data from [12]. 
agreement between analytical and numerical results. As expected, the Gaussian approximation is not exact, but to see deviations in a simulation, about $10^{5}$ samples have to be used. Analytically, corrections can be calculated: They are of order $R^{\prime \prime \prime}\left(0^{+}\right)^{4}$ and small. Physically, the distribution is narrower than a Gaussian.

\section{Anisotropic depinning, directed percolation, branching and all that}

We have discussed in section 13 isotopic depinning, which as the name suggest is a situation, where the system is invariant under a tilt. This isotropy can be broken through an additional anharmonic elasticity

$$
E_{\text {elastic }}=\int_{x} \frac{1}{2}[\nabla u(x)]^{2}+c_{4}[\nabla u(x)]^{4},
$$

leading to a drastically different universality class, the so-called anisotropic depinning universality class, as found recently in numerical simulations [30]. It has been observed in simulations [31,32], that the drift-velocity of an interface is increased, which can be intepreted as a tilt-dependent term, leading to the equation of motion in the form

$$
\partial_{t} u(x, t)=\nabla^{2} u(x, t)+\lambda[\nabla u(x, t)]^{2}+F(x, u(x, t))+f .
$$

However it was for a long time unclear, how this new term (proportional to $\lambda$ ), which usually is refered to as a KPZterm, is generated, especially in the limit of vanishing drift-velocity. In [5] we have shown that this is possible in a non-analytic theory, due to the diagram given in figure 13.

For anisotropic depinning, numerical simulations based on cellular automaton models which are believed to be in the same universality class [33,34], indicate a roughness exponent $\zeta \approx 0.63$ in $d=1$ and $\zeta \approx 0.48$ in $d=2$. On a phenomenological level it has been argued [33-35] that configurations at depinning can be mapped onto directed percolation in $d=1+1$ dimensions, which yields indeed a roughness exponent $\zeta_{\mathrm{DP}}=\nu_{\perp} / \nu_{\|}=0.630 \pm 0.001$, and it would be intriguing to understand this from a systematic field theory.

This theory was developed in [5], and we review the main resulsts here. A strong simplification is obtained by going to the Cole-Hopf transformed fields

$$
Z(x, t):=\mathrm{e}^{\lambda u(x, t)} \quad \Leftrightarrow \quad u(x, t)=\frac{\ln (Z(x, t))}{\lambda} .
$$

The equation of motion becomes after multiplying with $\lambda Z(x, t)$ (dropping the term proportional to $f$ )

$$
\partial_{t} Z(x, t)=\nabla^{2} Z(x, t)+\lambda F\left(x, \frac{\ln (Z(x, t))}{\lambda}\right) Z(x, t)
$$

and the dynamical action (after averaging over disorder)

$$
\mathcal{S}=\int_{x t} \tilde{Z}(x, t)\left(\partial_{t}-\nabla^{2}\right) Z(x, t)-\frac{\lambda^{2}}{2} \int_{x t t^{\prime}} \tilde{Z}(x, t) Z(x, t) \Delta\left(\frac{\ln Z(x, t)-\ln Z\left(x, t^{\prime}\right)}{\lambda}\right) \tilde{Z}\left(x, t^{\prime}\right) Z\left(x, t^{\prime}\right)
$$

This leads to the FRG flow equation at 1-loop order

$$
\begin{aligned}
\partial_{\ell} \Delta(u)= & (\epsilon-2 \zeta) \Delta(u)+\zeta u \Delta^{\prime}(u)-\Delta^{\prime \prime}(u)(\Delta(u)-\Delta(0))-\Delta^{\prime}(u)^{2} \\
& +2 \lambda \Delta(u) \Delta^{\prime}\left(0^{+}\right)+2 \lambda^{2}\left(\Delta(u)^{2}+\Delta(u) \Delta(0)\right)
\end{aligned}
$$

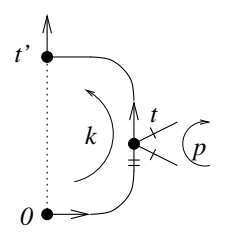

Figure 13: The diagram generating the irreversible nonlinear KPZ term with one disorder vertex and one $c_{4}$ vertex (the bars denote spatial derivatives). 


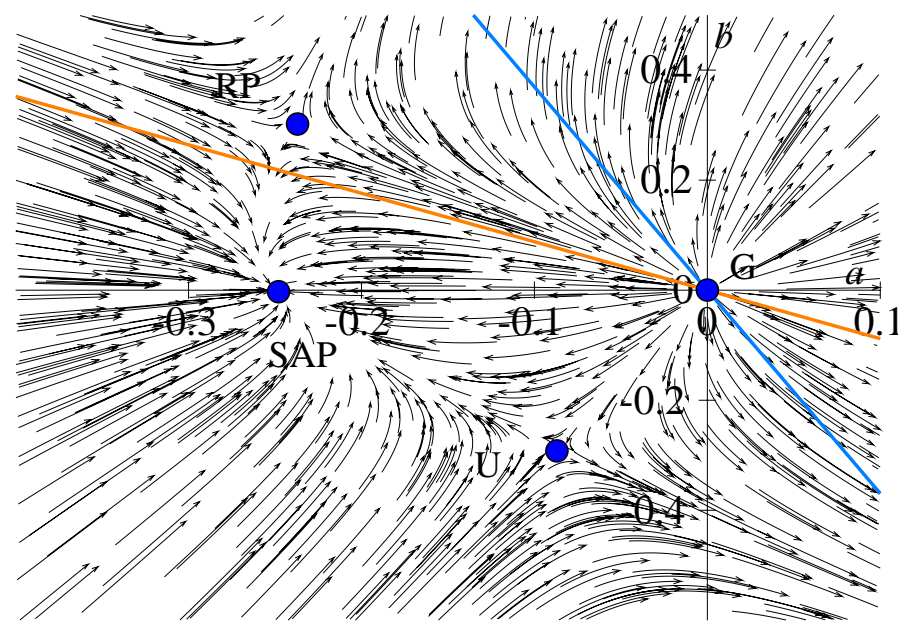

Figure 14: Fixed point structure for $\lambda=2$, which is a typical value. The ratio $c / b$ is not renormalized, see (15.9)-(15.10), such that $c / b$ is a parameter, fixed by the boundary conditions, especially $\lambda$. The fixed points are Gaussian G, Random Periodic RP (the generalization of the RP fixed point for $\lambda=0$ ), Self-Avoiding Polymers SAP, and Unphysical U.

The first line is indeed equivalent to (6.1) using $\Delta(u)=-R^{\prime \prime}(u)$. The second line is new and contains the terms induced by the KPZ term, i.e. the term proportional to $\lambda$ in (15.2).

Equation (15.6) possesses the following remarkable property: A three parameter subspace of exponential functions forms an exactly invariant subspace. Even more strikingly, this is true to all orders in perturbation theory [5]! The subspace in question is $(0 \leq u \leq 1 / \lambda)$

$$
\Delta(u)=\frac{\epsilon}{\lambda^{2}}\left(a+b \mathrm{e}^{-\lambda u}+c \mathrm{e}^{\lambda u}\right)
$$

The FRG-flow (15.6) closes in this subspace, leading to the simpler 3-dimensional flow:

$$
\begin{aligned}
\partial_{\ell} a & =a+4 a^{2}+4 a c+4 b c \\
\partial_{\ell} b & =b(1+6 a+b+5 c) \\
\partial_{\ell} c & =c(1+6 a+b+5 c)
\end{aligned}
$$

This flow has a couple of fixed points, given on figure 14. They describe different physical situations. The only globally attractive fixed point is SAP, describing self-avoiding polymers. This fixed point is not attainable from the physically relevant inital conditions, which lie (as fixed point RP) between the two separatrices given on figure 14 . All other fixed points are cross-over fixed points.

In the Cole-Hopf representation, it is easy to see why the exponential manifold is preserved to all orders. Let us insert (15.7) in (15.5). The complicated functional disorder takes a very simple polynomial form [5].

$$
\mathcal{S}=\int_{x t} \tilde{Z}(x, t)\left(\partial_{t}-\nabla^{2}\right) Z(x, t)-\int_{x} \int_{t<t^{\prime}} \tilde{Z}(x, t) \tilde{Z}\left(x, t^{\prime}\right)\left(a Z(x, t) Z\left(x, t^{\prime}\right)+b Z(x, t)^{2}+c Z\left(x, t^{\prime}\right)^{2}\right) .
$$

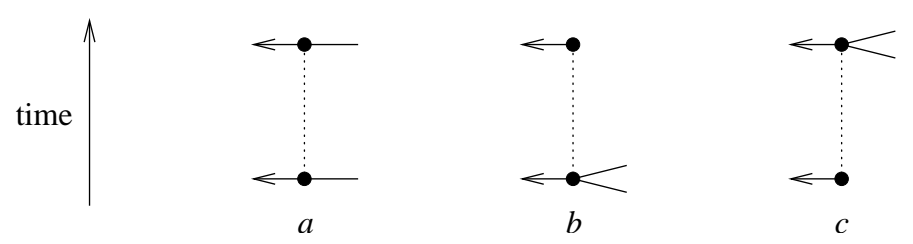

Figure 15: The three vertices proportional to $a, b$ and $c$ in equation (15.11). 
The vertices are plotted on figure 15. It is intriguing to interprete them as particle interaction $(a)$ and as different branching processes $(b$ and $c): Z$ destroys a particle and $\tilde{Z}$ creates one. Vertex $b$ can e.g. be interpreted as two particles coming together, annihilating one, except that the annihilated particle is created again in the future. However, if the annihilation process is strong enough, the reappearance of particles may not play a role, such that the interpretation as particle annihilation or equivalently directed percolation is indeed justified.

One caveat is in order, namely that the fixed points described above, are all crossover fixed point, and nothing can a priori be said about the strong coupling regime. However this is the regime seen in numerical simulations, for which the conjecture about the equivalence to directed percolation has been proposed. Thus albeit intriguing, the above theory is only the starting point for a more complete understanding of anisotropic depinning. Probably, one needs another level of FRG, so as standard FRG is able to treat directed polymers, or equivalently the KPZ-equation in the absence of disorder.

\section{Perspectives}

More interesting problems have been treated by the above methods, and much more remains to be done. We have applied our techniques to the statics at 3-loop order [6] and to the random field problem [9]. An expansion in $1 / N$, (by now we have obtained the effective action [7]), should allow to finally describe such notorious problems as the strong-coupling phase of the Kardar-Parisi-Zhang equation. Many questions are still open. Some have already been raised in these notes, another is wether FRG can also be applied to spin-glasses. We have to leave this problem for future research and as a challenge for the reader to plunge deeper into the mysteries of functional renormalization.

\section{Acknowledgements}

It is a pleasure to thank the organizers of TH 2002 for the opportunity to give this lecture. The results presented here have been obtained in a series of inspiring ongoing collaborations with Pierre Le Doussal, and I am grateful to him and my other collaborators Pascal Chauve, Werner Krauth and Alberto Rosso for all their enthousiasm and dedicated work. Numerous discussions with Leon Balents, Edouard Brézin and Andreas Ludwig are gratefully acknowledged.

\section{References}

[1] P. Chauve, P. Le Doussal and K.J. Wiese, Renormalization of pinned elastic systems: How does it work beyond one loop?, Phys. Rev. Lett. 86 (2001) 1785-1788, cond-mat/0006056.

[2] P. Le Doussal and K.J. Wiese, Functional renormalization group at large $N$ for random manifolds, Phys. Rev. Lett. 89 (2002), cond-mat/0109204v1.

[3] P. Le Doussal, K.J. Wiese and P. Chauve, 2-loop functional renormalization group analysis of the depinning transition, Phys. Rev. B 66 (2002) 174201, cond-mat/0205108.

[4] P. Le Doussal, K.J. Wiese and P. Chauve, Two loop FRG study of pinned manifolds, in preparation.

[5] P. Le Doussal and K.J. Wiese, Functional renormalization group for anisotropic depinning and relation to branching processes, Phys. Rev. E 67 (2003) 016121, cond-mat/0208204.

[6] K.J. Wiese and P. Le Doussal, 3-loop FRG study of pinned manifolds, in preparation.

[7] P. Le Doussal and K.J. Wiese, The functional renormalization group approach to a manifold in disorder: large $N$, in preparation.

[8] P. Le Doussal and K.J. Wiese, 2-loop functional renormalization group treatment of pinned elastic manifolds in $N$ dimensions, in preparation. 
[9] P. Le Doussal and K.J. Wiese, 2-loop functional renormalization group treatment of random field models, in preparation.

[10] P. Le Doussal and K.J. Wiese, The functional renormalization group approach to a manifold in disorder: $1 / N$ expansion, in preparation.

[11] P. Le Doussal and K.J. Wiese, Higher correlations, universal distributions and finite size scaling in the field theory of depinning, cond-mat/0301465 (2003).

[12] A. Rosso, W. Krauth, P. Le Doussal, J. Vannimenus and K.J. Wiese, Universal interface width distributions at the depinning threshold, cond-mat/0301464 (2003).

[13] K.J. Wiese, Disordered systems and the functional renormalization group: A pedagogical introduction, Acta Physica Slovaca 52 (2002) 341, cond-mat/0205116.

[14] S. Lemerle, J. Ferré, C. Chappert, V. Mathet, T. Giamarchi and P. Le Doussal, Domain wall creep in an Ising ultrathin magnetic film, Phys. Rev. Lett. 80 (1998) 849.

[15] S. Moulinet, C. Guthmann and E. Rolley, Roughness and dynamics of a contact line of a viscous fluid on a disordered substrate, Eur. Phys. J. A 8 (2002) 437-43.

[16] K.B. Efetov and A.I. Larkin, Sov. Phys. JETP 45 (1977) 1236.

[17] A.I. Larkin, Sov. Phys. JETP 31 (1970) 784.

[18] G. Parisi and N. Sourlas, Random magnetic fields, supersymmetry, and negative dimensions, Phys. Rev. Lett. 43 (1979) 744-5.

[19] F.J. Wegner and A. Houghton, Renormalization group equation for critical phenomena, Phys. Rev. A 8 (1973) 401-12.

[20] D.S. Fisher, Interface fluctuations in disordered systems: $5-\epsilon$ expansion, Phys. Rev. Lett. 56 (1986) $1964-97$.

[21] L. Balents, private communication.

[22] L. Balents and D.S. Fisher, Large- $N$ expansion of $4-\varepsilon$-dimensional oriented manifolds in random media, Phys. Rev. B 48 (1993) 5949-5963.

[23] A.A. Middleton, Numerical results for the ground-state interface in a random medium, Phys. Rev. E 52 (1995) R3337-40.

[24] M. Mézard and G. Parisi, Replica field theory for random manifolds, J. Phys. I (France) 1 (1991) 809-837.

[25] L. Balents, J.P. Bouchaud and M. Mézard, The large scale energy landscape of randomly pinned objects, Journal de Physique I 6 (1996) 1007-20.

[26] M. Kardar, G. Parisi and Y.-C. Zhang, Dynamic scaling of growing interfaces, Phys. Rev. Lett. 56 (1986) 889-892.

[27] E. Marinari, A. Pagnani and G. Parisi, Critical exponents of the KPZ equation via multi-surface coding numerical simulations, J. Phys. A 33 (2000) 8181-92.

[28] T. Nattermann, S. Stepanow, L.H. Tang and H. Leschhorn, Dynamics of interface depinning in a disordered medium, J. Phys. II France 2 (1992) 1483-1488.

[29] O. Narayan and D.S. Fisher, Threshold critical dynamics of driven interfaces in random media, Phys. Rev. B 48 (1993) 7030-42. 
[30] A. Rosso and W. Krauth, Origin of the roughness exponent in elastic strings at the depinning threshold, Phys. Rev. Lett. 87 (2001) 187002.

[31] L.A.N. Amaral, A. L. Barabasi and H.E. Stanley, Critical dynamics of contact line depinning, Phys. Rev. Lett. 73 (1994).

[32] L.-H. Tang, M. Kardar and D. Dhar, Driven depinning in anisotropic media, Phys. Rev. Lett. 74 (1995) 920-3.

[33] L.-H. Tang and H. Leschhorn, Pinning by directed percolation, Phys. Rev. A 45 (1992) R8309-12.

[34] S.V. Buldyrev, A.-L. Barabasi, F. Caserta, S. Havlin, H.E. Stanley and T. Vicsek, Anomalous interface roughening in porous media: experiment and model, Phys. Rev. A 45 (1992) R8313-16.

[35] S.C. Glotzer, M.F. Gyure, F. Sciortino, A. Coniglio and H.E. Stanley, Pinning in phase-separating systems, Phys. Rev. E 49 (1994) 247-58. 